\section{Clinical findings and diagnosis in 84 cats with nasal disease after undergoing advanced diagnostic imaging}

\section{Ned Kuehn, Jessica Romine, Whitney Hough, Sean Teichner, Michelle Rose}

BluePearl Veterinary Partners - Michigan, Southfield, MI, USA

Medical records and advanced diagnostic imaging results were reviewed in 84 cats over an 8-year period. Nasal computed tomography (CT) was performed on 82 of the cats and magnetic resonance imaging (MRI) on 2 cats. The study population comprised 49 male and 35 female cats with a mean age of 9.94 years and mean weight of $4.57 \mathrm{~kg}$. The breeds most represented were 53 Domestic short and long hair cats, 6 Siamese, 4 Maine Coon, and 3 Ocicat.

Presenting clinical signs were highly variable with 61 (73\%) having acute or chronic purulent nasal discharge, 35 (42\%) sneezing, 34 (40\%) obstructive nasal breathing, 19 (23\%) epistaxis, and 13 (15\%) facial asymmetry or exophthalmia. Common CT/MRI findings were 32 (38\%) turbinate destruction, $21(25 \%)$ unilateral or bilateral bulla opacification, 13 (15\%) vomer deviation, 12 (14\%) septal destruction, 7\% (8\%) cribriform plate destruction, 7 (8\%) ocular displacement, and
5 (6\%) hard palate destruction.

Within the study population, 42 (50\%) had chronic rhinitis, 37 (44\%) neoplasia, 2 nasopharyngeal polyp, and 1 each with parasitic, fungal, and foreign body rhinosinusitis. Sarcomas accounted for $55 \%$ and adenocarcinomas for $45 \%$ of cats with biopsy confirmed nasal neoplasia $(n=31)$. The majority of sarcomas were lymphosarcoma (88\%) and the majority of carcinomas were adenocarcinoma (93\%). Upon re-review of the CT/MRI studies, 3 of 45 cats with a biopsy diagnosis of chronic rhinitis were identified as most likely to have nasal neoplasia.

There was no significant difference in body weight or sex of cats with nasal disease. The mean age of cats with chronic rhinitis (8.76 years) was younger than those with neoplasia (12.0 years) $(p=0.0010)$. There was no difference in age between those having lymphosarcoma or adenocarcinoma. There was no significant difference in age between cats having lymphosarcoma to those having chronic rhinitis; however, cats with adenocarcinomas were significantly older compared to those with chronic rhinitis $(p=0.0016)$.

Clinical signs of epistaxis and facial asymmetry or exophthalmia, and CT/MRI findings of septal, cribriform, or hard palate destruction were highly correlated but not specific for neoplasia. CT/MRI was extremely effective in determining the extent of sinonasal disease and discriminating between advanced neoplastic diseases from those possibly having chronic rhinosinusitis. However, the $\mathrm{CT}$ findings with nasal lymphoma may mimic those seen with chronic rhinitis, while fungal rhinosinusitis may mimic the destructive bony changes seen with advanced neoplasia.

\section{A retrospective questionnaire based study of Pneumonia in the Irish Wolfhound}

\section{Angela Bodey}

Abbey House Veterinary Hospital, Morley, Leeds, UK

The Irish Wolfhound Health Group considers that pneumonia is overrepresented in Irish wolfhounds (IWHs), that it can be severe and recurrent, and is often fatal. Reports of respiratory syndromes in IWHs are found in the literature, but there is no comprehensive study concerning incidence or epidemiological factors for the UK cohort.

The aim of this study was to gather information about clinical cases of acute respiratory disease in IWHS, using an owner questionnaire. The questionnaire was designed to be simple to complete, covering management of disease, with veterinary records where available, and details of outcome.

The age range of affected dogs was 3 months to 10 years (median age 5 years), including seventeen entire and seven neutered females, twenty three entire and six neutered males.

From the narrative description of clinical signs in the questionnaire, animals were allocated a severity score from 0 to 5 for each of seven clinical signs (nasal discharge, inappetance, recumbency, pyrexia, cough, tachypnoea, extended neck). A score of 0 indicated that sign was absent; numbers 1 to 5 were used to describe the severity of the sign, considering degree and duration.

Severity scores were added to give each animal a total clinical score. Thirty one had a total clinical score of more than 10 but less than 20, and amongst these seven died (23\%). Eighteen had a total clinical score of 20 or more, and amongst these eight died (44\%). There were no fatalities for animals with a total clinical score of 10 or less.

Affected dogs received a range of treatments, including various antibiotics, NSAIDs, mucolytics, physiotherapy and supportive care (intravenous fluid therapy, turning). Twenty one of the animals received three or more antibiotics, sixteen received two antibiotics and sixteen received a single antibiotic. In particular, seventeen animals received cetiofur, of which six died (35\%), and thirty six did not receive cetiofur, and which nine died (25\%).

Kennel cough was described by primary vets prior to a diagnosis of pneumonia in six animals; three (50\%) died. One dog was documented to have megoesophagus, and this dog died.

Recurrent pneumonia was seen in twenty one dogs (40\%). Of these, two had an owner reported link with primary ciliary dyskinesia, and one had diagnosed (and surgically managed) laryngeal paralysis. 